\title{
Quantitative Chemical Phase Imaging Using Backscattered Electron Imagery, A New Approach
}

\author{
Edward P. Vicenzi, ${ }^{* * *}$ Andrew R. Konicek, ${ }^{* *}$, and Catherine M. Corrigan $* * *$ \\ * Museum Conservation Institute, Smithsonian Institution, Suitland, MD \\ ** Surface and Microanalysis Science Division, NIST, Gaithersburg, MD \\ ***Department of Mineral Sciences, NMNH, Smithsonian Institution, Washington, DC
}

Motivation/Initial Specimen Suite: During the 2003 field season to the Miller Range (MIL), the Antarctic Search for Meteorites (ANSMET) team recovered MIL 03346, deemed to be Martian in origin [1]. This stone is a cumulate of pyroxene crystals $\left[(\mathrm{Mg}, \mathrm{Fe}) \mathrm{CaSi}_{2} \mathrm{O}_{6}\right]$ with rare olivine $\left[(\mathrm{Mg}, \mathrm{Fe})_{2} \mathrm{SiO} 4\right]$ grains. The crystal size of both minerals are on the order of 100 's of micrometers in diameter and are set in a fine-grained matrix, down to the submicrometer scale, where multiple phases are complexly intergrown. Three new specimens (MIL 090030, 090032, and 090136) found in 2009 share a number of petrographic features with MIL 03346 and therefore these stones are thought to be derived from the same parent meteorite [2]. Quantifying the microstructural relationship between these four specimens has served as the motivation for a new image processing routine to measure chemical phase abundance and distribution using backscattered electrons (BSE).

Description of Method: In order to rigorously distinguish chemical phases and measure their volume, we have developed a method for rapidly analyzing polished samples using BSE imagery. Our initial sample suite requires a mosaic of tiled high-pixel density images (2048 x 1768, 2 mm $\mathrm{HFW}$ ) to maximize the area obtained for the coarse-grained portion of the microstructure. BSE detector contrast is optimized using an accelerating voltage of $13-30 \mathrm{kV}$, an energy range ensuring the BSE signal response is linearly related to average atomic number $(\bar{Z})$. Histograms for the images are automatically fitted with a series of Gaussian peaks through the use of a series of fits within a narrow range of image contrast until all gray levels have been analyzed (Fig. 1a). These Gaussians are then assigned a color and "traced-back" to the image where the corresponding pixels retain the peak's false color. Fitting only major histogram features in the fit residuals ensures that the solution is not over determined by an unnecessarily large number of Gaussians. The integrated counts associated with each peak represent either a mineral phase, or a distinct chemistry within a compositionally zoned phase (Fig. 2a-e). This method accounts for pixels where peaks overlap one another owing to similar $\bar{Z}$ by way of peak area scaling (Fig. 1b). This rigorous deconvolution greatly improves the accuracy of quantitative phase imaging relative to standard BSE region-ofinterest mapping. Efforts to enhance the contrast sensitivity of typical 8 bit gray level images by use of differing dwell times, frame integrations, and increased bit depth have been explored.

Independently determined chemical phase images derived from hyperspectral X-ray data cubes of fine-structured regions in the meteorites provide a lower level of fidelity relative to the high pixel density of the quantitative BSE phase imaging results (Fig. 3 a \& b).

\section{References}

[1] Satterwhite and Righter, 2004, Antarctic Meteorite Newsletter 27, 1-2

[2] McKay and Schwandt, 2005, Lunar and Planetary Science Conference 36, \#2351 

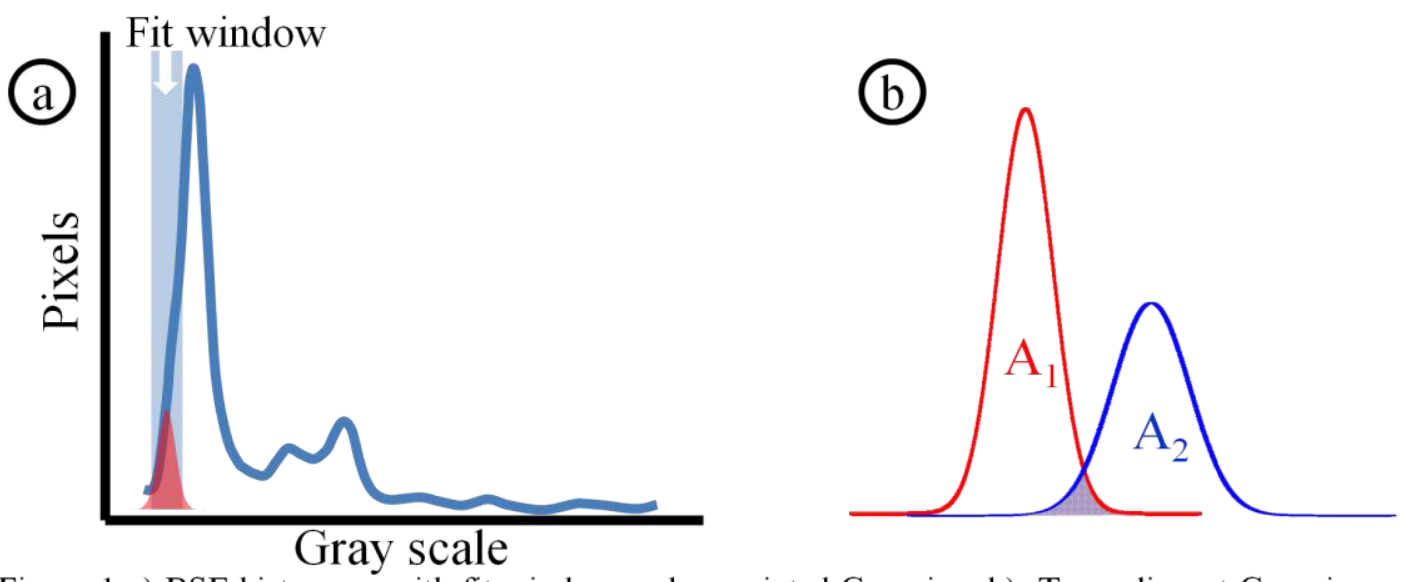

Figure 1 a) BSE histogram with fit window and associated Gaussian. b) Two adjacent Gaussian peaks where the overlap region is partitioned according to the ratio of the areas of the peaks.
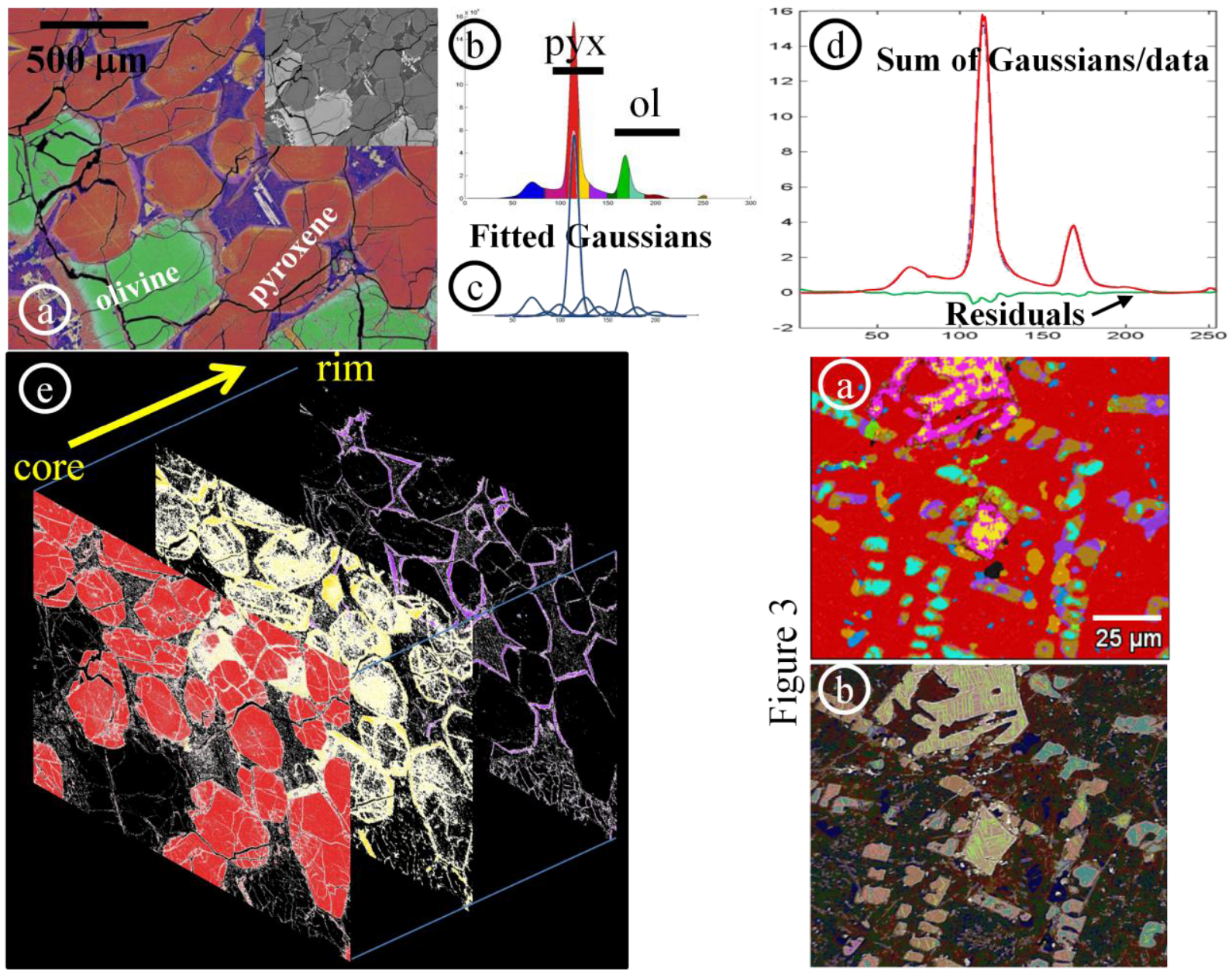

Figure 2 a) False-colored BSE phase image based upon histogram deconvolution of MIL 090136 image (inset: gray scale BSE). b) False-colored intensity histogram. c) fitted Gaussian peaks. d) Histogram (black), sum of the Gaussians (red), and fitting residuals (green) for the image in (a). e) Three phase masks for pyroxene representing core crystal regions of lower avg. $Z$ (red) through rim compositions of higher avg. $Z$ (lavender).

Figure 3 a) Phase image of fine scale microstructure derived from a hyperspectral X-ray data cube $(256 \times 220$ pixels). b) Phase image of same region derived from deconvolved BSE histogram (2048 x 1768 pixels). 